\title{
Precalificación de una conexión soldada a momento viga-columna para aplicaciones en edificios metálicos
}

\author{
Hernán M. Acero* ${ }^{\S}$ Patricia Guerrero* \\ * Escuela de Ingeniería Civil y Geomática, Universidad del Valle, Cali, Colombia \\ §e-mail:hera202@hotmail.com
}

(Recibido: Octubre 4 de 2005 - Aceptado: Agosto 18 de 2006)

\begin{abstract}
Resumen
La precalificación de la conexión a momento viga-columna está basada en las consideraciones de la norma Provisiones sísmicas para edificios metálicos de acero AISC-2002. En esta norma se propone el procedimiento estándar para la realización de pruebas cíclicas a las conexiones, con el fin de comprobar su comportamiento ante solicitaciones dinámicas. Este ensayo de precalificación se realizó experimentalmente en el marco de pruebas del laboratorio de estructuras de la Universidad del Valle y se evaluó matemáticamente mediante un modelo de elementos finitos. En este modelo se consideró el comportamiento elasto-plástico del acero con endurecimiento cinemático. Los resultados del modelo se compararon con los resultados experimentales. El procedimiento de diseño y el detallado de la conexión fueron exitosamente validados tanto por el ensayo experimental de precalificación como por la simulación en elementos finitos.
\end{abstract}

Palabras clave: Ensayo cíclico de precalificación, Provisiones sísmicas, Ángulo de deriva de entrepiso, Esfuerzo de fluencia, Momento plástico, Rótula plástica, Elementos finitos.

\section{Prequalification of a beam-to-column moment connection for steel building applications}

\begin{abstract}
The prequalification of the beam-to-column moment connection is based on the AISC-2002 standard Seismic provisions for structural steel buildings. In this norm, a standard procedure is proposed for undertaking cyclic tests of connections, with the aim of checking their behavior under dynamic solicitations. This prequalification test was carried out in the test-frame of the structure laboratory at Universidad del Valle, and it was mathematically evaluated by use of a finite element model that considered the elastic-plastic behavior of kinematically-hardened steel. The results from the mathematical model were compared with experimental data. The design procedure and the details of the connection were successfully validated by means of an experimental prequalification test and a finite element simulation.
\end{abstract}

Keywords: Prequalification cyclic tests, Seismic provisions, Inter-story drift angle, Yield stress, Plastic moment, Plastic hinge, Finite elements. 


\section{Introducción}

En investigaciones recientes de conexiones a momento viga-columna, se ha encontrado una diferencia entre el esfuerzo de fluencia real $F_{y}$ y el esfuerzo de fluencia especificado $F_{y e}$ para el acero de mayor utilización: ASTM A36. La fluencia real del material puede alcanzar a ser un $50 \%$ mayor que la especificada (FEMA-355E, 2000). Esta diferencia es la causa principal de las fallas en el comportamiento de las conexiones. Por esta razón, se debe garantizar la permanencia elástica en los elementos conectados. Para lograr esto, es necesario diseñar las conexiones de modo que se tenga una resistencia superior al momento plástico de las vigas que se conectan.

En muchos países, el diseño de las conexiones se está realizando de acuerdo a parámetros que han sido previamente estandarizados, precalificándolas por su buen comportamiento durante ensayos. Por lo tanto, es importante que en el país se desarrolle y adopte una tecnología propia para el diseño de las conexiones, ya que las normas internacionales presentan cambios, entre los cuales está que todas las conexiones del sistema de resistencia sísmico de estructuras de acero que no hayan sido precalificadas, deben ser probadas en el laboratorio. De ahí la importancia de precalificar las conexiones.

Los requisitos para precalificar una conexión, para uso en pórticos resistentes a momento (con capacidad especial de disipación de energía), son las siguientes: deberá ser capaz de soportar un ángulo de deriva de entrepiso de no menos de $0.04 \mathrm{rad}$ y la resistencia a flexión de la conexión efectuada en la cara de la columna debe ser como mínimo el $80 \%$ del momento plástico nominal de la viga (AISC, 2002).

Los criterios para diseño de las conexiones vigacolumna en Colombia, si se usan las especificaciones de la Norma Colombiana de Diseño y Construcciones Sismo-resistentes (NSR-98), presentan un detallado similar al mostrado en la Figura 1 (FEMA-355D, 2000). La aleta de la viga se une a la aleta de la columna con soldadura de penetración. La placa de cortante se une a la aleta de columna con soldadura y transfiere los esfuerzos al alma de la viga por

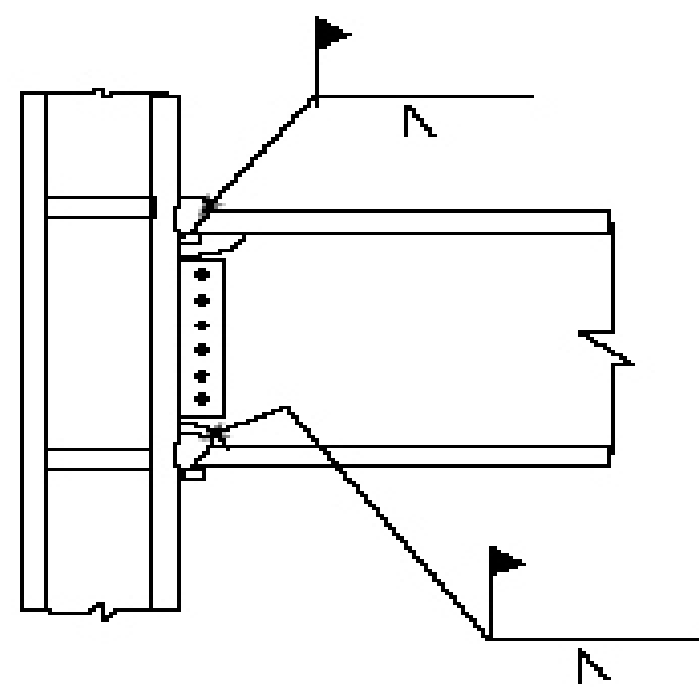

Figura 1. Conexión soldada resistente a momento utilizada antes de 1994 (Fema-355D, 2000).

medio de pernos. Las placas de continuidad se sueldan a la columna, ayudando a prevenir daños en la aleta y en el alma de la columna. Este detallado mostró fallas frágiles durante los sismos de Northridge en 1994 y Kobe en 1995. Surge la necesidad de proponer tipos de conexiones vigacolumna que, respaldadas con ensayos experimentales, garanticen un comportamiento dúctil de la estructura durante un evento sísmico.

\section{Metodología}

\subsection{Diseño del espécimen}

En las Figuras 2 y 3 se muestra el prototipo de conexión escogida para este proyecto. Esta es de tipo cubre placas, para edificios con altura entre 8 y 10 pisos.

La conexión tiene una ranura en la cubre placa superior (Garza et al., 2004) de $60 \mathrm{~mm}$ de ancho $\left(b_{f b} / 3\right)$, la cual se usó para aplicar soldadura plana horizontal en filete, conectando la aleta de la viga con la cubre placa y evitando así la soldadura sobre cabeza. Se sabe que este tipo de soldadura requiere de soldadores muy calificados, dada la dificultad en la aplicación. Además es un proceso lento y costoso por lo que se debe evitar esta posición siempre que sea posible (McCormac, 1996). 


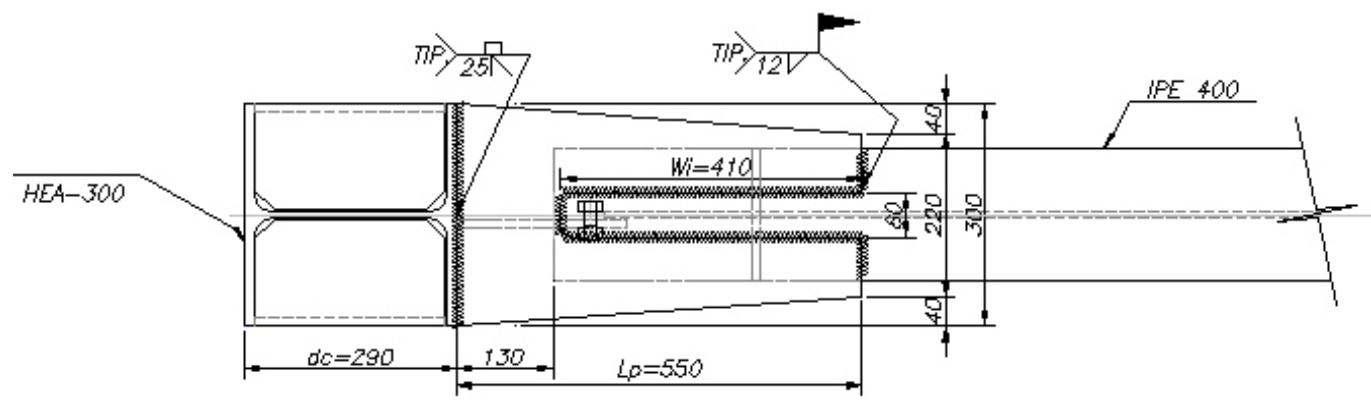

Figura 2. Vista superior del prototipo ensayado.

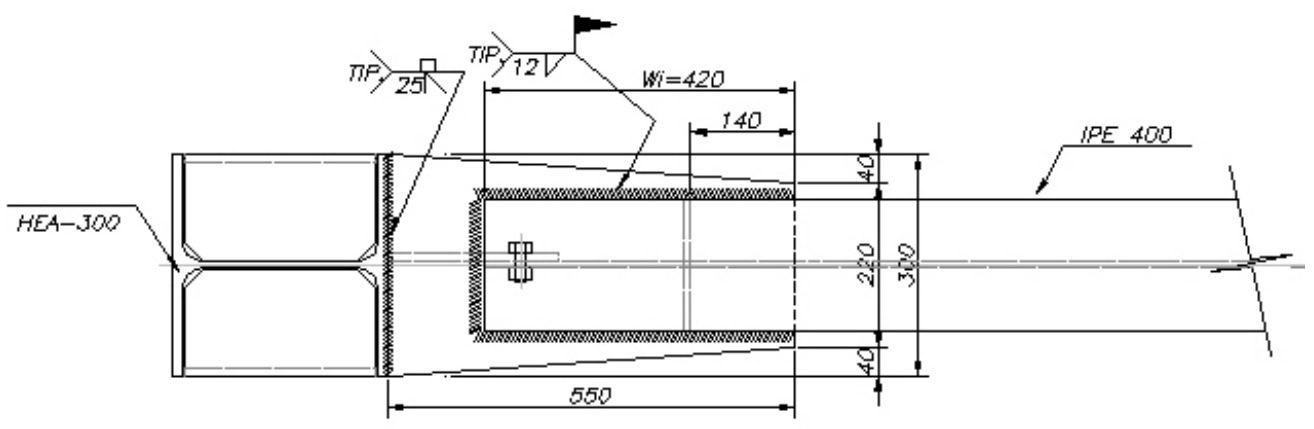

Figura 3. Vista inferior del prototipo ensayado.

Para facilitar el montaje, la conexión tiene dos pernos de $\Phi 5 / 8$ " grado 5 (Figuras 2 y 3 ) que unen la placa de cortante y el alma de la viga y que se pueden remover después de aplicada la soldadura definitiva. Estas condiciones hacen que la conexión sea segura y económica.

Además, la conexión tiene platinas dobles igualmente espaciadas y platinas de continuidad en la zona del panel. Con estos elementos se evitan las deformaciones causadas por las concentraciones de esfuerzos en este sitio.

Las propiedades geométricas del espécimen se presentan en la Tabla 1. La Tabla 2, muestra las propiedades de los materiales y la Tabla 3, las propiedades mecánicas de la soldadura.

Tabla 1. Dimensiones de los perfiles y placas.

\begin{tabular}{lll}
\hline \multicolumn{1}{c}{ Descripción } & \multicolumn{1}{c}{ Viga IPE400 } & Columna HEA300 \\
\hline Ancho de la aleta, $\mathrm{mm}$ & $b_{f b}=180$ & $b_{f c}=300$ \\
Espesor de la aleta, $\mathrm{mm}$ & $t_{f b}=13.5$ & $t_{f c}=14$ \\
Espesor del alma, mm & $t_{w b}=8.6$ & $t_{w c}=8.5$ \\
Profundidad, mm & $d_{b}=400$ & $d_{c}=290$ \\
Módulo plástico de sección, $\mathrm{mm}^{3}$ & $Z_{b x}=1.307 \times 10^{6}$ & $Z_{\mathrm{cx}}=1.383 \times 10^{6}$ \\
& $Z_{b y}=2.29 \times 10^{5}$ & $Z_{\mathrm{cy}}=6.41 \times 10^{5}$ \\
Área, $\mathrm{mm}^{2}$ & $A_{b}=8450$ & $A_{c}=11300$ \\
Momento de inercia, $\mathrm{mm}^{4}$ & $I_{b x}=2.313 \times 10^{8}$ & $I_{c x}=1.826 \times 10^{8}$ \\
Longitud, mm & $I_{b y}=1.318 \times 10^{7}$ & $I_{c y}=6.310 \times 10^{7}$ \\
Placa a cortante, mm & $L_{b}=3800$ & $L_{c}=3000$ \\
Cubre placa superior e & $h_{s t}=314$ & $t_{s t}=12$ \\
inferior, mm & $L_{s t}=230$ & \\
\hline
\end{tabular}


Tabla 2. Propiedades mecánicas de los materiales.

\begin{tabular}{lcccc}
\hline \multicolumn{1}{c}{ Descripción } & $\begin{array}{c}\text { Tensión de fluencia } \\
F_{y}(\mathrm{MPa})\end{array}$ & $\begin{array}{c}\text { Tensión ultima } \\
F_{u}(\mathrm{MPa})\end{array}$ & $\begin{array}{c}\text { Elongación } \\
(\%)\end{array}$ & Acero \\
\hline Valores nominales & 250 & 400 & 21 & A36 \\
Columna HEA300 & 296 & 447 & 28 & A36 \\
Alma & 271 & 433 & 33 & A36 \\
Aleta & 345 & 468 & 26 & A36 \\
Viga IPE400 & 294 & 440 & 27 & A36 \\
Alma & & & & \\
Aleta & 320 & 414 & 34 & A36 \\
Cubre placa & & & & \\
Platina 25 mm & & & & \\
\hline
\end{tabular}

(Laboratorio ASTIM-SENA, Regional Valle, Ensayo a tracción ASTM 370, Informes No. EM 020104 , EM 024804).

Tabla 3. Propiedades mecánicas de la soldadura.

\begin{tabular}{lc}
\hline \multicolumn{2}{c}{ Soldadura E 7018} \\
\hline Resistencia a la tracción (M Pa ) & $510-590$ \\
Límite de fluencia (M Pa ) & $430-510$ \\
Elongación (\%) & $24-36$ \\
Reducción del área (\%) & $50-75$ \\
Resistencia al impacto Charpy en $\mathrm{V} \mathrm{a}-29^{\circ} \mathrm{C}(\mathrm{J})$ & 70 \\
\hline
\end{tabular}

A continuación se presentan algunos criterios que se tienen en cuenta en el diseño de la conexión:

- Momento plástico de la viga:

$$
M_{p}=Z_{x b} F_{y b}
$$

donde $M_{p}$ es el momento plástico de la viga, $Z_{x b}$ es el módulo plástico de la sección de la viga en el que se encuentra localizada la rotula plástica y $F_{y b}$ es la mínima tensión de fluencia de la viga.

- Momento plástico esperado de la viga:

$$
M_{p r}=C_{p r} R_{y} M_{p}
$$

donde $M_{p r}$ es el momento plástico esperado de la viga, $C_{p r}$ es el factor que tiene en cuenta la resistencia máxima de la conexión, incluyendo el endurecimiento por tensión, restricciones locales, refuerzo adicional y otras condiciones de la conexión y $R_{y}$ es el coeficiente obtenido de la norma Provisiones Sísmicas del AISC-2002, y que relaciona la tensión de fluencia esperada con la mínima tensión de fluencia especificada de la viga. Se usó un valor de 1.2 para $C_{p r}$ (FEMA-350, 2000). Para acero $\mathrm{A} 36, R_{y}=1.5$.

- La conexión debe cumplir la condición de columna fuerte-viga débil y una manera de probar esto es mediante la siguiente condición (Ec. 9.3 de AISC, 2002):

$$
\frac{\sum M_{p c}^{*}}{\sum M_{p b}^{*}}>1
$$

donde $\sum M_{p c}^{*}$ es la sumatoria de los momentos en la columna por encima y por debajo del nudo de la intersección de las líneas centrales de las vigas y columnas y $\sum M_{p b}^{*}$ es la sumatoria de momento(s) 
en la(s) viga(s) en la intersección de las líneas centrales de vigas y columnas.

- Se deben usar platinas de continuidad de las aletas de la viga a través del alma de la columna, cuando el espesor de la aleta de la columna es menor que el dado por las siguientes condiciones (Ec. 3-5 y Ec. 3-6, FEMA-350, 2000):

$$
\begin{aligned}
& t_{f c}<0.4\left[1.8 b_{f b} t_{f b} \frac{F_{y b} R_{y b}}{F_{y c} R_{y c}}\right]^{1 / 2} \\
& t_{f c}<\frac{b_{f b}}{6}
\end{aligned}
$$

donde $t_{f c}$ es el mínimo espesor requerido de la aleta de columna cuando las platinas de continuidad no son provistas, $b_{f b}$ es el ancho del aleta de la viga (para nuestro caso, es el ancho de la cubre placa $\left.b_{p}\right), t_{f b}$ es el espesor de la aleta de la viga (para nuestro caso, es el espesor de la cubre placa $\left.\mathrm{t}_{p}\right), F_{y b}\left(F_{y c}\right)$ es la mínima tensión de fluencia de la aleta de la viga (columna) y $R_{y b}\left(R_{y c}\right)$ es el coeficiente que relaciona la tensión de fluencia esperada con la mínima tensión de fluencia especificada de la viga (columna).

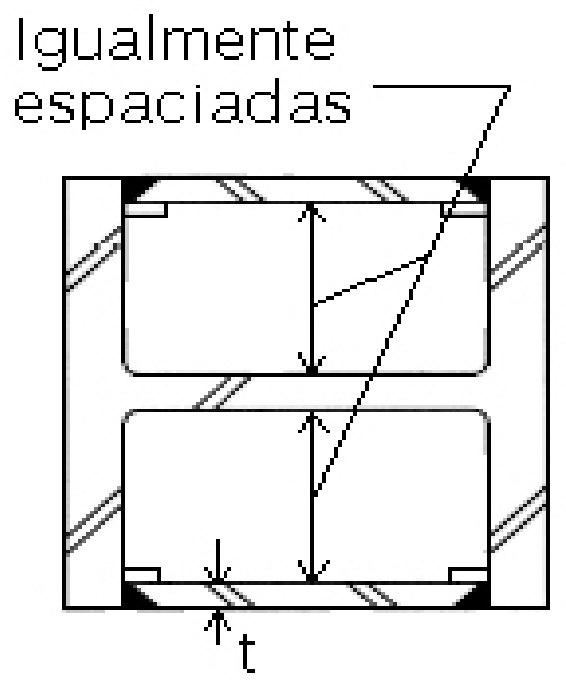

Figura 4. Platinas dobles en zona del panel.
- En la Figura 4 se muestra la disposición de las platinas dobles en la zona del panel y debe cumplirse con la condición siguiente (Ec. 9.2 de AISC 2002):

$$
t \geq \frac{d_{z}+w_{z}}{90}
$$

donde $d_{z}$ es la altura de la zona del panel entre las platinas de continuidad y $w_{z}$ es el ancho de la zona del panel entre las aletas de la columna.

- La resistencia de diseño a corte $\varnothing_{v} R_{v}$ de la zona del panel se determina con la siguiente ecuación (Ec. 9.1 de AISC 2002), usando un valor $\varnothing_{v}=1$ :

$$
\varnothing_{v} R_{v}=0.6 F_{y} d_{c} t
$$

sujeta a la condición:

$$
1+\frac{3 b_{f c} t_{f c}^{2}}{d_{b} d_{c} t} \geq \frac{M_{f}}{d_{b}}
$$

donde $\varnothing_{v} R_{v}$ es la resistencia de diseño a corte de la zona del panel, $d_{c}$ es la altura total de la columna, $t$ es el espesor total de la zona del panel que incluye las platinas dobles, $b_{f c}$ es el ancho de la aleta de la columna, $t_{f c}$ es el espesor de la aleta de la columna, $d_{b}$ es la altura total de la viga y $M_{f}$ es el momento plástico en la cara de la columna.

\subsection{Historia de carga}

El prototipo del modelo matemático en elementos finitos y el del espécimen en el marco de pruebas para la evaluación experimental, se ensayaron con una historia de carga, como se muestra en la Figura 5 y en la Tabla 4.

Esta historia se basa en los requerimientos del Apéndice S de la norma Provisiones Sísmicas del AISC-2002. La historia de carga consiste en incrementos moderados de ciclos de deformación, controlando el ángulo de deriva de entrepiso. 


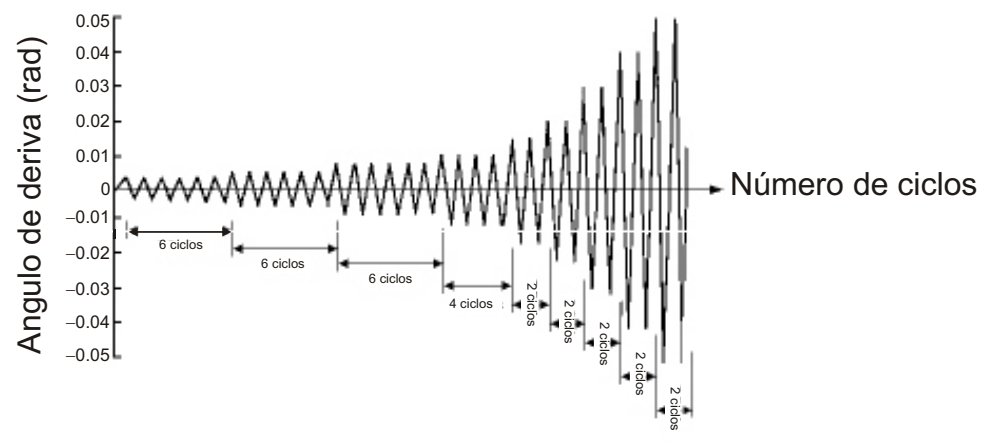

Figura 5. Grafica estándar de la historia de carga (Apéndice S, Provisiones Sísmicas de AISC-2002).

Tabla 4. Historia de carga.

\begin{tabular}{cccc}
\hline Paso de carga \# & Deformación pico, $\theta(\mathrm{rad})$ & Número de ciclos, $\mathrm{n}$ & $\delta(\mathrm{mm})$ \\
\hline 1 & 0.00375 & 6 & 14.25 \\
2 & 0.005 & 6 & 19.00 \\
3 & 0.0075 & 6 & 28.50 \\
4 & 0.01 & 4 & 38.00 \\
5 & 0.015 & 2 & 57.00 \\
6 & 0.02 & 2 & 76.00 \\
7 & 0.03 & 2 & 114.00 \\
8 & 0.04 & 2 & 152.00 \\
\hline
\end{tabular}

\subsection{Análisis por elementos finitos}

Con este método se evaluó el comportamiento de la conexión. Se determinaron las zonas donde se presentarían altas concentraciones de tensiones y deformaciones durante el ensayo cíclico. Con esta información se identificaron los sitios óptimos para colocar la instrumentación y el mecanismo de falla esperado del prototipo. Para la simulación del prototipo definitivo en elementos finitos, se consideró un modelo elasto-plástico del material con endurecimiento cinemático. Se usaron 74516 elementos en total, donde 71569 fueron elementos brick y 2947 fueron elementos shell.

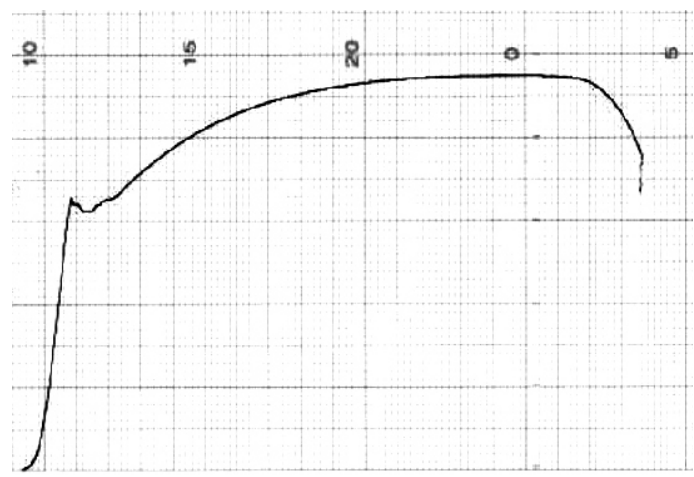

Figura 6. Curva esfuerzo-deformación de aleta viga IPE 400. 
En la Figura 6 se muestra la curva esfuerzo vs. deformación de la aleta de la viga, obtenida en ensayo experimental a tracción con la norma ASTM 370. Se realizaron dos ensayos para aleta, alma y cubre placa. Los valores que definen la curva se usaron como datos de entrada de las propiedades del material durante el proceso de cómputo del software usado (Algor).

En la Figura 7 se observa el modelo de elementos finitos del prototipo de la conexión mostrando la rótula plástica, la cual se generó en la viga.

En la Tabla 5 se muestra el desempeño de la viga de la conexión en los últimos ciclos de la simulación. Se observa que la viga tiene un comportamiento elástico hasta el ciclo 22 (0.01 rad).
Después, el comportamiento en la aleta pasa a ser plástico, continuando con el alma hasta el ciclo 30 $(0.04 \mathrm{rad})$ y finalmente falla.

\subsection{Fabricación del espécimen}

Una vez realizado el análisis del prototipo por elementos finitos, se procedió a fabricar el espécimen que posteriormente fue ensayado en el marco de pruebas con una instrumentación acorde a los resultados teóricos. En esta sección se describe este ensayo y se comparan los resultados con los obtenidos mediante elementos finitos.

El espécimen de prueba fue fabricado en la planta de la empresa $B \& S$ Engineering, siguiendo la práctica convencional.

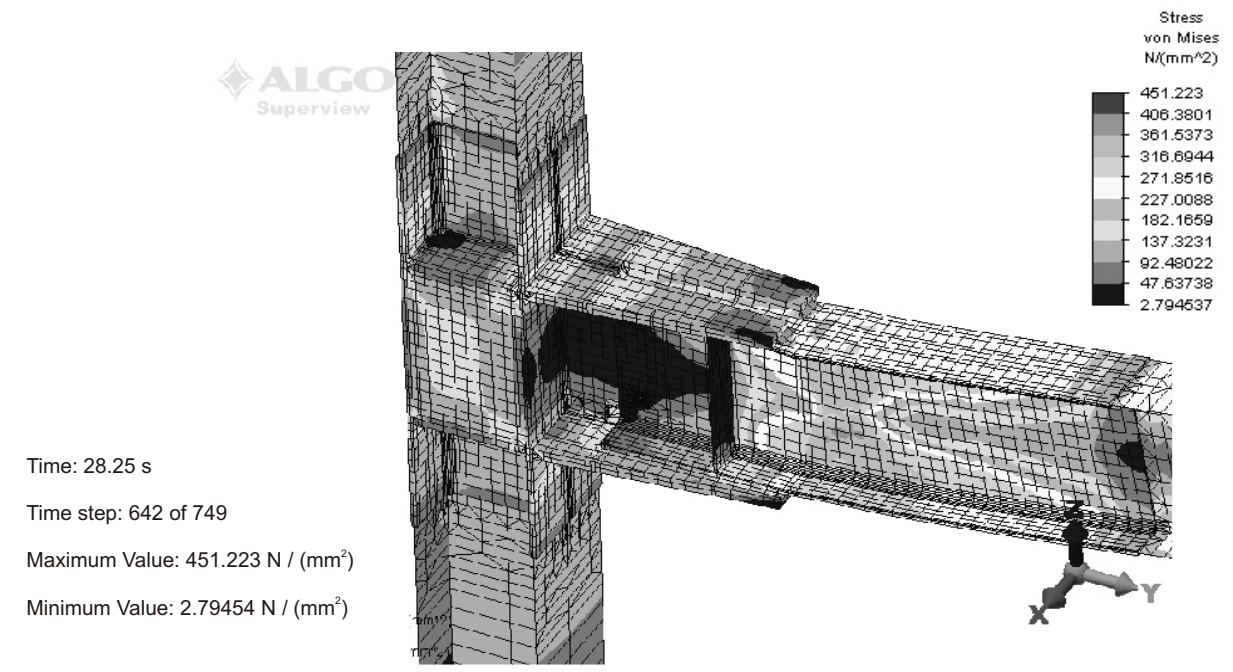

Figura 7. Prototipo de la conexión en elementos finitos.

Tabla 5. Comportamiento de la viga en la zona de la rotula plástica.

\begin{tabular}{|c|c|c|c|c|c|c|c|c|c|c|}
\hline \multirow[t]{2}{*}{$\begin{array}{l}\text { Rotación } \theta \\
\text { (rad) }\end{array}$} & \multirow[t]{2}{*}{$\begin{array}{c}\text { Tiempo } \\
\text { (seg) }\end{array}$} & \multirow[t]{2}{*}{ Ciclo } & \multirow{2}{*}{$\begin{array}{c}\text { Esfuerzos } \\
\text { Von Mises (MPa) } \\
\text { elemento finito }\end{array}$} & \multicolumn{2}{|c|}{$\begin{array}{l}F_{y}(\mathrm{MPa}) \\
\text { Viga IPE400 }\end{array}$} & \multicolumn{2}{|c|}{$\begin{array}{c}F_{u}(\mathrm{MPa}) \\
\text { Viga IPE400 }\end{array}$} & \multirow[t]{2}{*}{ Elástico } & \multirow[t]{2}{*}{ Plástico } & \multirow[t]{2}{*}{ Falla } \\
\hline & & & & aleta & alma & aleta & alma & & & \\
\hline 0.01 & 21.25 & 22 & 287.22 & 294 & 345 & 440 & 468 & $\mathrm{X}$ & & \\
\hline 0.01 & 21.75 & 22 & 287.30 & 294 & 345 & 440 & 468 & $\mathrm{X}$ & & \\
\hline 0.015 & 22.25 & 23 & 341.75 & 294 & 345 & 440 & 468 & & $\mathrm{X}$ & \\
\hline 0.015 & 22.75 & 23 & 341.43 & 294 & 345 & 440 & 468 & & $\mathrm{X}$ & \\
\hline 0.03 & 27.75 & 28 & 358.84 & 294 & 345 & 440 & 468 & & $\mathrm{X}$ & \\
\hline 0.04 & 28.25 & 29 & 452.22 & 294 & 345 & 440 & 468 & & $\mathrm{X}$ & \\
\hline 0.04 & 28.75 & 29 & 438.98 & 294 & 345 & 440 & 468 & & $\mathrm{X}$ & \\
\hline 0.04 & 29.25 & 30 & 646.55 & 294 & 345 & 440 & 468 & & & $\mathrm{X}$ \\
\hline 0.04 & 29.75 & 30 & 968.32 & 294 & 345 & 440 & 468 & & & $\mathrm{X}$ \\
\hline Eje neutro & 30 & ---- & 443.78 & 294 & 345 & 440 & 468 & & & $\mathrm{X}$ \\
\hline
\end{tabular}


Las cubre placas, placa de cortante y los elementos que hacen parte de la zona del panel fueron soldadas en el taller. Para formar la unión de la conexión viga-columna, la soldadura fue también hecha en la planta en condiciones parecidas a las condiciones reales de montaje de una estructura en el campo : el espécimen fue soldado en posición vertical, simulando todas las posiciones de soldadura y obstáculos encontrados en la práctica estándar.
La soldadura fue realizada por personal calificado, y fueron examinadas por un inspector capacitado en forma visual y usando la prueba de tintas. Se usó soldadura de penetración (unión de las cubre placas y columna, platinas de continuidad y platinas dobles en zona del panel) y soldadura en filete (para el resto de la conexión), la soldadura se aplicó de acuerdo a la norma D1.1 / D1.1M de American Welding Society (AWS, 2004).
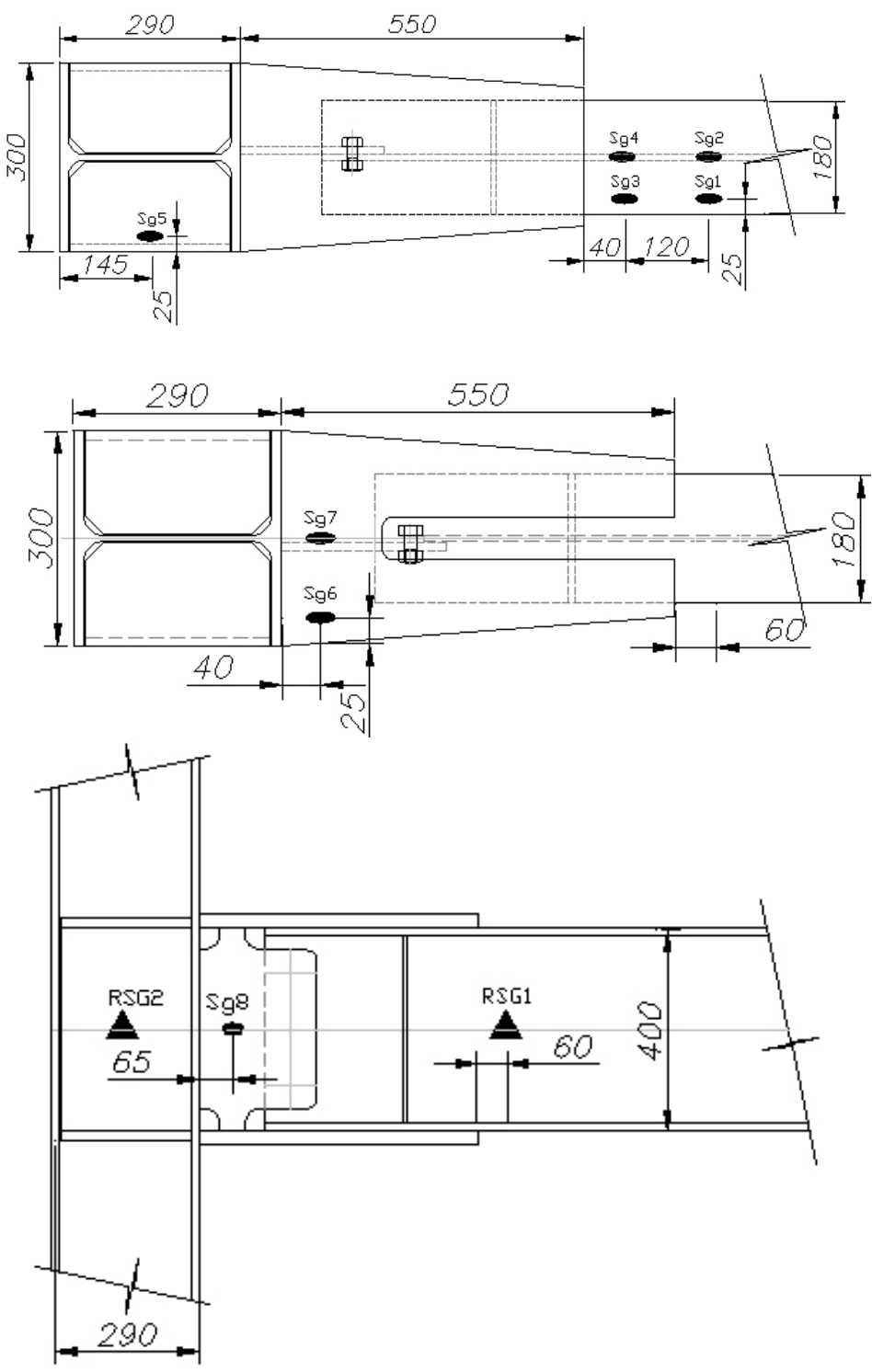

Figura 8. Instrumentación del espécimen de prueba. 

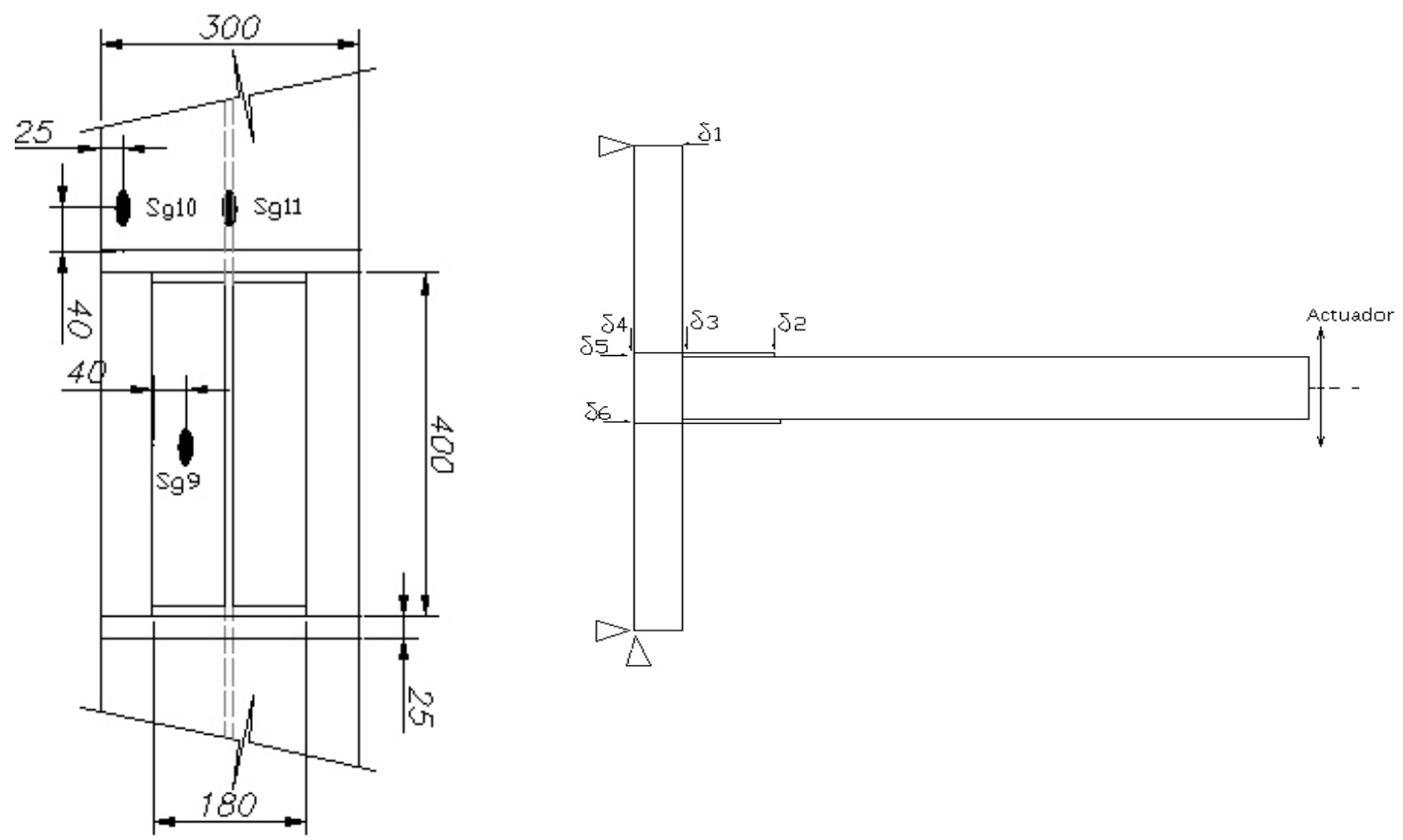

Figura 8. (continuación)

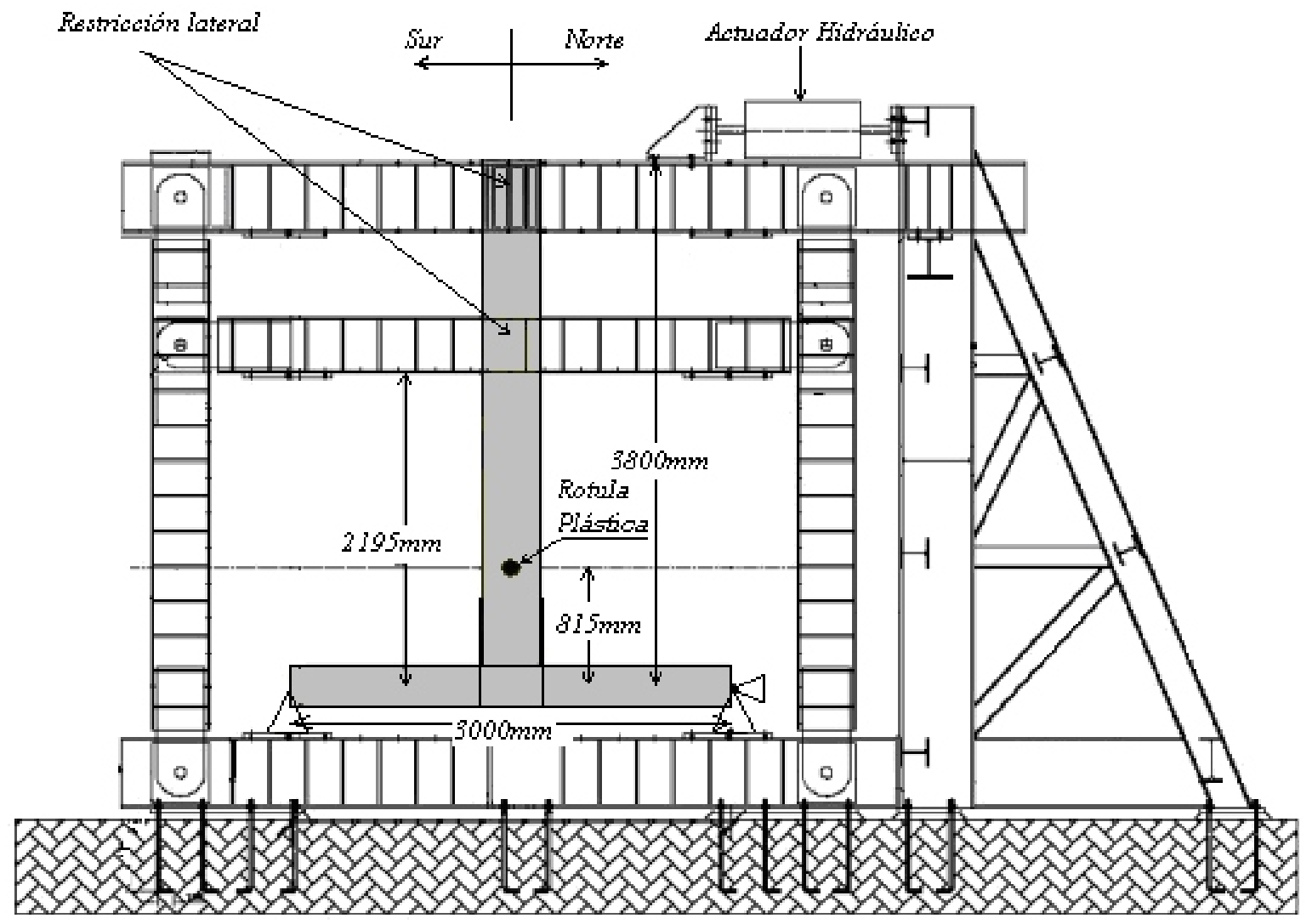

Figura 9. Detalle del montaje del ensayo. 


\subsection{Instrumentación del ensayo}

En la Figura 8 se muestra la disposición de la instrumentación del espécimen. Los extensómetros (LVDT), se usaron para medir los desplazamientos $(\delta)$, y con esta información se determinaron las rotaciones. Las galgas $\left(S_{\mathrm{g}}\right)$ y las rosetas (RSG) extensométricas se usaron para medir las deformaciones unitarias y se obtuvieron las tensiones generadas durante la prueba.

\subsection{Montaje del ensayo}

En la Figura 9 se muestra el montaje del espécimen de prueba. Este, en forma de $\mathrm{T}$, es considerado como una viga en voladizo conectada a la aleta de la columna por medio de cubre placas. El espécimen fue rotado $90^{\circ}$ con respecto a su posición en una construcción y colocado entre el marco de pruebas. La columna es pernada en sus partes finales al pórtico de carga y el extremo libre de la viga fue conectado mediante un pasador al actuador hidráulico, con el que se aplicó la carga cíclica.

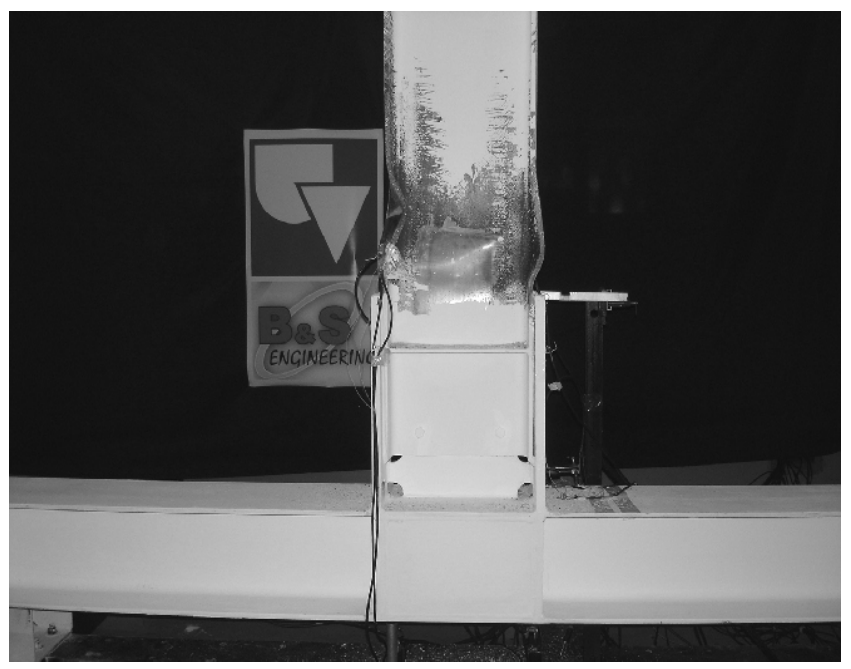

Experimental

Time: $30 \mathrm{~s}$

Time step: 749 of 749

Maximum Value: $443.779 \mathrm{~N} /\left(\mathrm{mm}^{2}\right)$

Minimum Value: $1.45446 \mathrm{~N} /\left(\mathrm{mm}^{2}\right)$

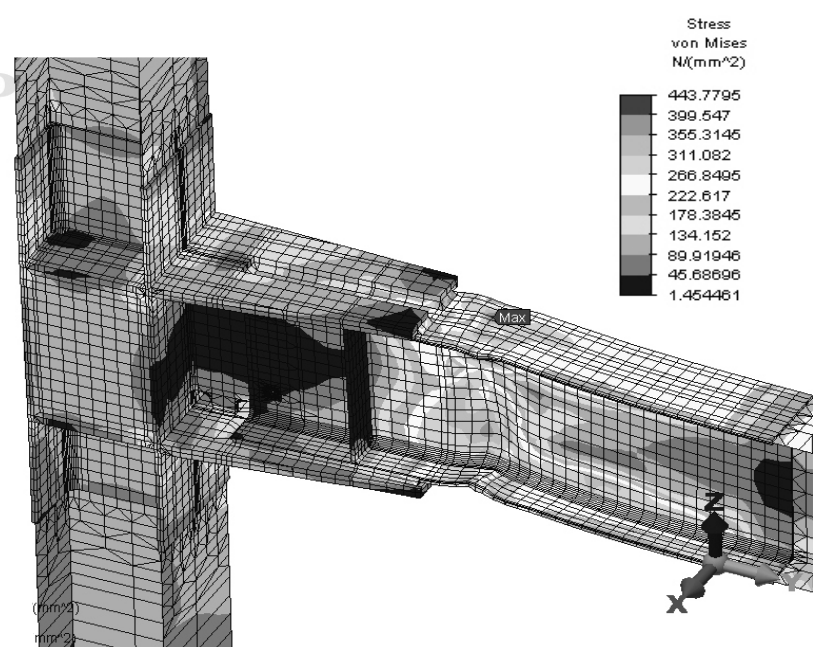

Elementos finitos

Figura 10. Rótula plástica en la viga. 
Esta posición horizontal de la columna en la parte del marco, paralela al piso del laboratorio, es óptima, porque permite libre acceso a ambas caras de la viga. Además, la cercanía al piso del laboratorio hace que la instrumentación y las medidas de seguridad sean fáciles de implementar.

Las dimensiones y las condiciones de soporte del espécimen fueron escogidas para representar los puntos de inflexión teóricas de la viga y columna en una típica construcción de un pórtico.

\section{Resultados y discusión}

\subsection{Rótula plástica}

En la Figura 10 se aprecia la rótula plástica en la fotografía superior y su simulación por elementos finitos en el gráfico inferior. Se observa que la energía fue disipada por la fluencia cíclica de la viga, produciéndose la falla de la viga por pandeo local de las aletas y alma en la formación de la rótula plástica. No se presentó distorsión de la zona de panel.

Experimentalmente, la rótula se localizó a $815 \mathrm{~mm}$ del eje de la columna, con una longitud en zona de la rótula de $240 \mathrm{~mm}$. Para la simulación por elementos finitos, los valores son de $812 \mathrm{~mm}$ y $234 \mathrm{~mm}$, respectivamente. Estos valores son cercanos a lo estipulado en el capítulo 7.4 de la norma AISC-2002, en el cual la longitud de la zona de la rótula plástica se define como la mitad de la profundidad de la viga $(200 \mathrm{~mm})$.

En la Tabla 6 se muestra la secuencia de falla de la conexión. Esta secuencia se determina por comparación entre el valor del límite de fluencia

Tabla 6. Secuencia de falla de la conexión.

\begin{tabular}{|c|c|c|c|c|c|c|c|c|c|c|}
\hline \multirow[b]{2}{*}{$\begin{array}{l}\text { Punto } \\
\text { evaluado }\end{array}$} & \multirow[b]{2}{*}{ Descripción } & \multicolumn{3}{|c|}{$\begin{array}{l}\text { Resultados en simulación } \\
\text { Elementos Finitos }\end{array}$} & \multicolumn{3}{|c|}{$\begin{array}{l}\text { Resultados en ensayo } \\
\text { experimental }\end{array}$} & \multicolumn{2}{|c|}{$\begin{array}{l}\text { Ensayo de } \\
\text { materiales }\end{array}$} & \multirow[t]{2}{*}{$\begin{array}{l}\text { Secuencia } \\
\text { de falla }\end{array}$} \\
\hline & & Ciclo & $\begin{array}{c}\theta \\
(\mathrm{rad})\end{array}$ & $\begin{array}{l}\text { Esfuerzo } \\
\text { (MPa) }\end{array}$ & Ciclo & $\begin{array}{c}\theta \\
(\mathrm{rad})\end{array}$ & $\begin{array}{l}\text { Esfuerzo } \\
\text { (MPa) }\end{array}$ & $\begin{array}{c}F_{y} \\
(\mathrm{MPa})\end{array}$ & $\begin{array}{c}F_{u} \\
(\mathrm{MPa})\end{array}$ & \\
\hline $\mathrm{S}_{\mathrm{g} 1}$ & $\begin{array}{l}\text { Aleta viga } \\
\text { IPE400 }\end{array}$ & 23 & 0.015 & 296 & 25 & 0.020 & 294 & 294 & 440 & 2 \\
\hline $\mathrm{S}_{\mathrm{g} 2}$ & $\begin{array}{l}\text { Aleta viga } \\
\text { IPE400 }\end{array}$ & 23 & 0.015 & 300 & 24 & 0.015 & 302 & 294 & 440 & 2 \\
\hline $\mathrm{S}_{\mathrm{g} 3}$ & $\begin{array}{l}\text { Aleta viga } \\
\text { IPE400 }\end{array}$ & 19 & 0.010 & 312 & 20 & 0.010 & 336 & 294 & 440 & 1 \\
\hline $\mathrm{S}_{\mathrm{g} 4}$ & $\begin{array}{l}\text { Aleta viga } \\
\text { IPE400 }\end{array}$ & 19 & 0.010 & 294 & 20 & 0.010 & 315 & 294 & 440 & 1 \\
\hline $\mathrm{S}_{\mathrm{g} 5}$ & $\begin{array}{l}\text { Placa } \\
\text { continuidad }\end{array}$ & 27 & 0.030 & 68 & 32 & 0.050 & 85 & 290 & 421 & 4 \\
\hline$S_{g 6}$ & $\begin{array}{l}\text { Cubre placa } \\
\text { superior }\end{array}$ & 27 & 0.030 & 165 & 29 & 0.040 & 182 & 320 & 414 & 4 \\
\hline $\mathrm{S}_{\mathrm{g} 7}$ & $\begin{array}{l}\text { Cubre placa } \\
\text { superior }\end{array}$ & 27 & 0.030 & 200 & 30 & 0.040 & 44 & 320 & 414 & 4 \\
\hline $\mathrm{S}_{\mathrm{g} 8}$ & $\begin{array}{l}\text { Placa } \\
\text { cortante }\end{array}$ & 30 & 0.040 & 7 & 30 & 0.040 & 44 & [250] & {$[400]$} & 4 \\
\hline$S_{g 9}$ & $\begin{array}{l}\text { Atiesador } \\
\text { en viga }\end{array}$ & 30 & 0.040 & 7 & 32 & 0.050 & 9 & [250] & {$[400]$} & 4 \\
\hline $\mathrm{S}_{\mathrm{g} 10}$ & $\begin{array}{l}\text { Aleta columna } \\
\text { HEA300 }\end{array}$ & 25 & 0.020 & 265 & 29 & 0.040 & 285 & 271 & 433 & 4 \\
\hline $\mathrm{S}_{\mathrm{g} 11}$ & $\begin{array}{l}\text { Aleta columna } \\
\text { HEA300 }\end{array}$ & 25 & 0.020 & 180 & 27 & 0.030 & 178 & 271 & 433 & 4 \\
\hline $\mathrm{RSG}_{1}$ & $\begin{array}{l}\text { Alma viga } \\
\text { IPE400 }\end{array}$ & 30 & 0.040 & 340 & 32 & 0.050 & 362 & 345 & 468 & 3 \\
\hline $\mathrm{RSG}_{2}$ & $\begin{array}{l}\text { Platina doble } \\
\text { zona panel }\end{array}$ & 26 & 0.020 & 97 & 32 & 0.050 & 202 & [250] & {$[400]$} & 4 \\
\hline
\end{tabular}

Los valores entre corchetes son teóricos (Beer et al., 2003). 
Experimental

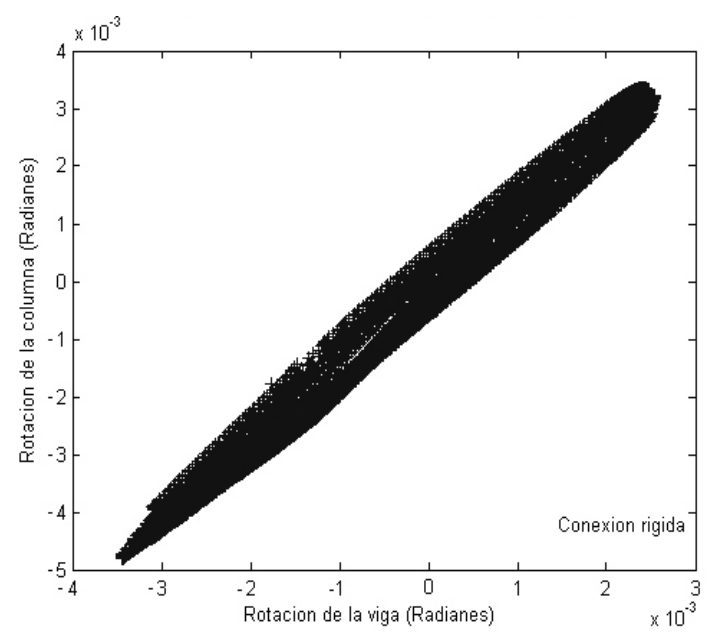

Elementos finitos

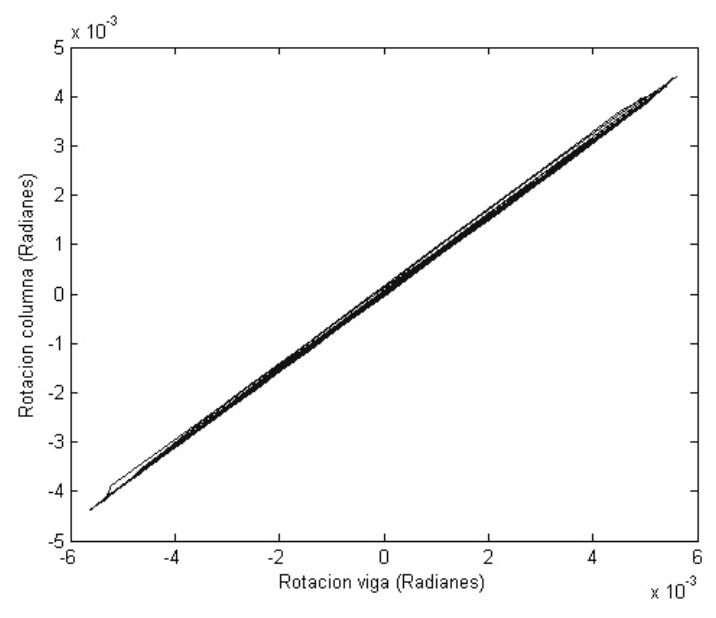

Figura 11. Rotación de la columna vs. rotación de la viga.
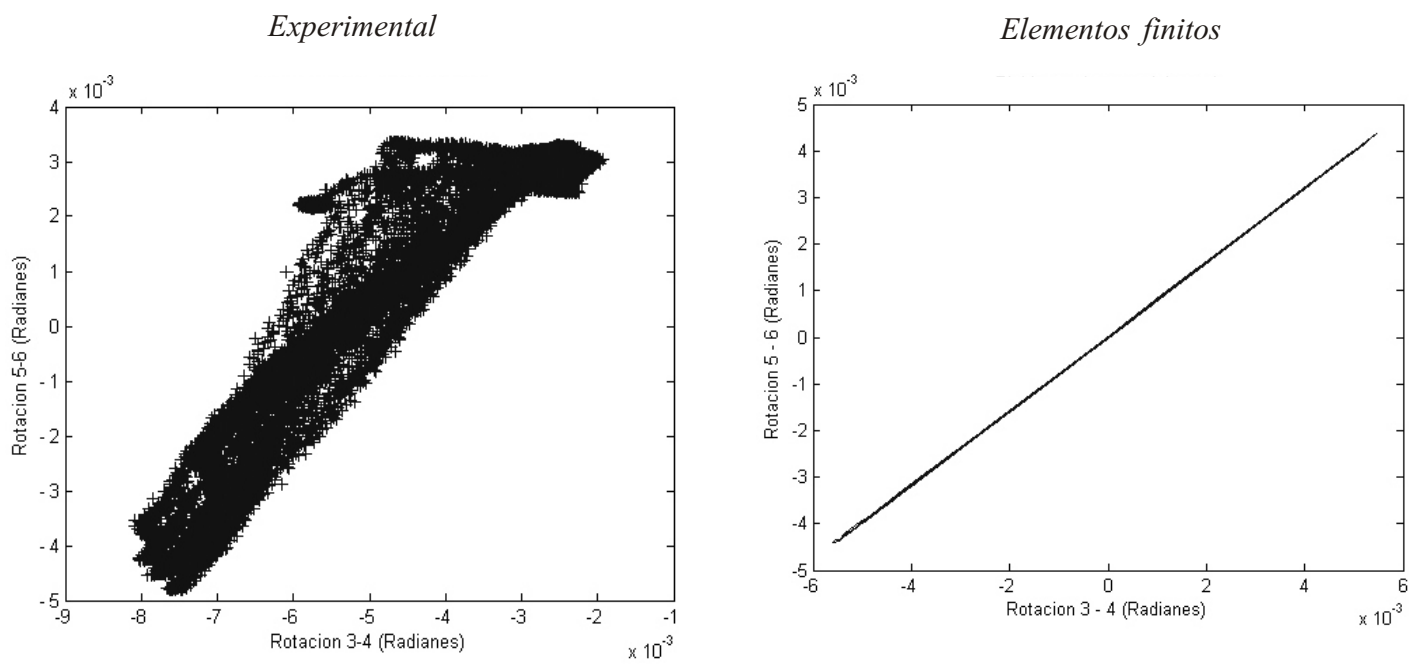

Figura 12. Rigidez en la zona del panel.

del material con los registros experimentales y con los de la simulación por elementos finitos. Por lo tanto, se define la falla como el punto donde el material empieza a fluir.

De acuerdo con la ubicación de las galgas (véase la Figura 8), la conexión falla inicialmente en la aleta de la viga donde se generó la rótula plástica (ver en la columna de secuencia de falla los números 1 y 2). Luego se propaga al alma de la viga (número 3). También se observa cómo los demás elementos de la conexión permanecieron en el rango elástico (número 4), indicando que no fallan. 


\subsection{Comportamiento de la conexión en zona del panel}

De acuerdo con la distribución de la Figura 11 (una pendiente constante) la conexión es rígida, ya que la deformación en la columna es similar a la de la viga en la zona del panel de la conexión. La rotación de la viga se calcula a partir de los desplazamientos $\delta_{2}$ y $\delta_{3}$ de la columna a partir de $\delta_{5}$ y $\delta_{6}$.
En la parte experimental y en la simulación en elementos finitos, se capturaron 14800 y 749 registros en cada sitio evaluado, respectivamente, presentándose una mayor densidad de puntos en el gráfico experimental.

Las gráficas de la Figura 12 muestran la rigidez de la zona de panel con valores muy pequeños, indicando que su distorsión angular es prácticamente nula.
Experimental

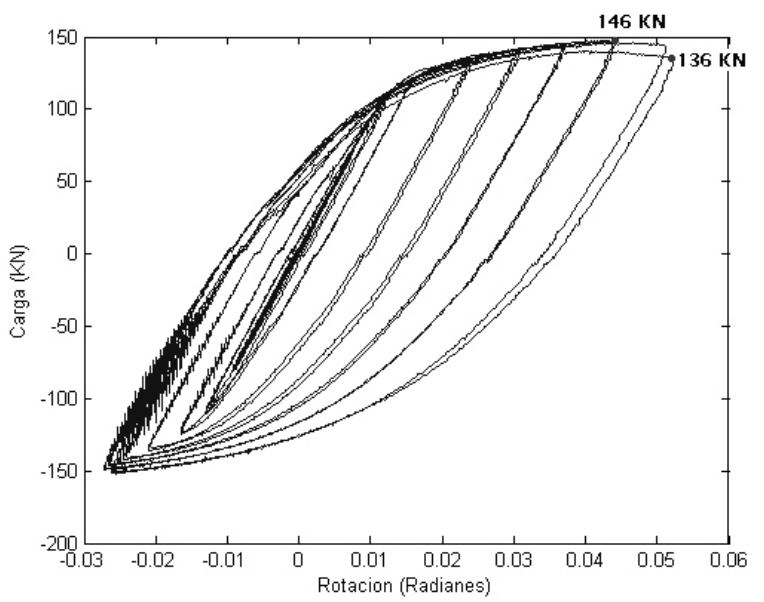

Elementos finitos

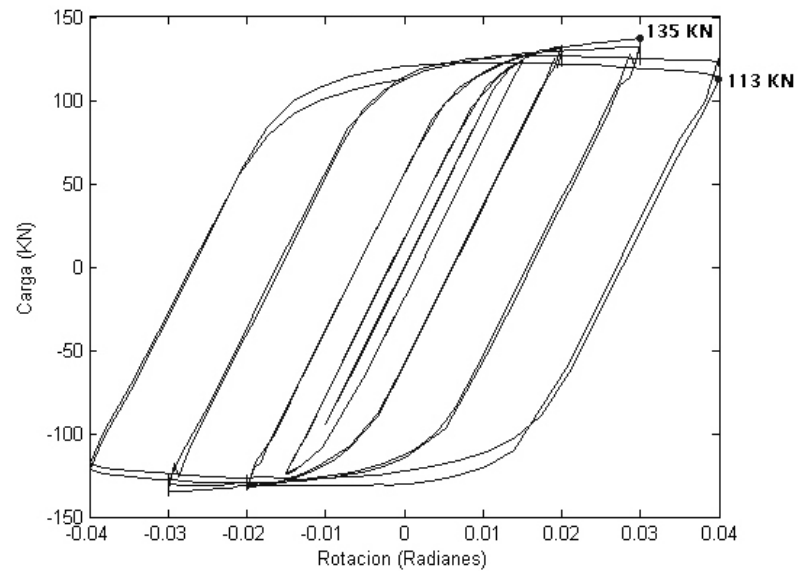

Figura 13. Gráfica histerética carga vs. rotación.

Experimental

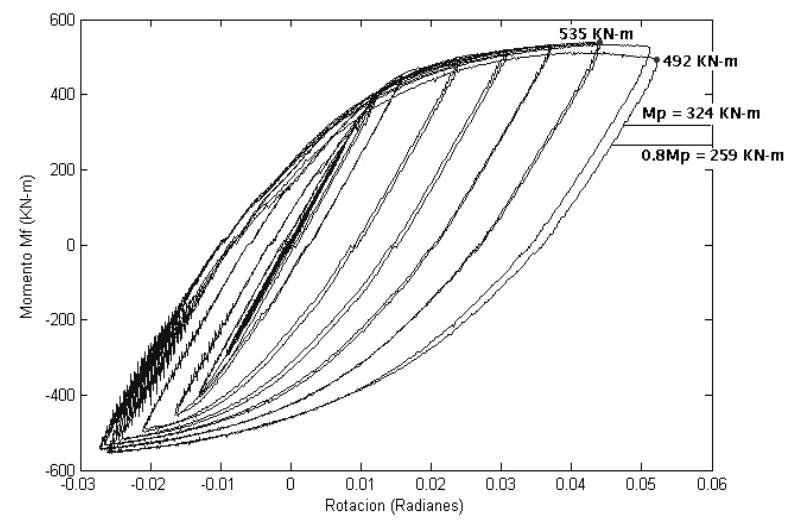

Elementos finitos

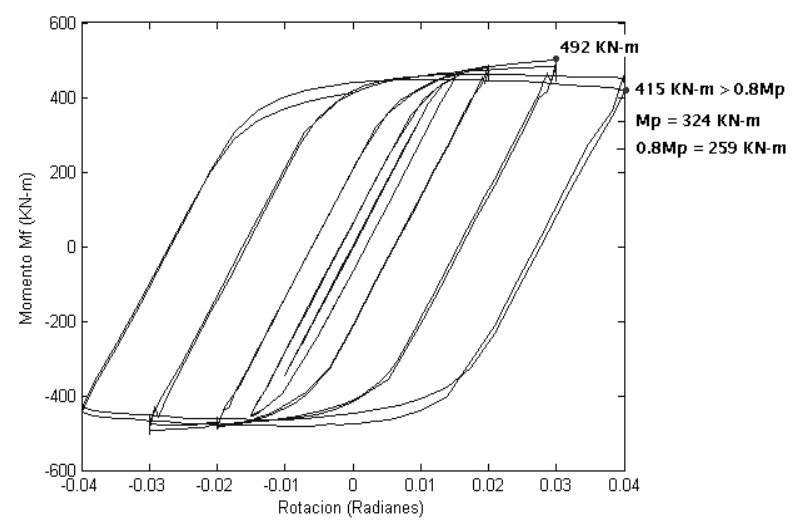

Figura 14. Gráfica histerética momento $M_{f}$ vs. rotación. 
Lo anterior demuestra que el panel no contribuye a la deformación de la conexión y que toda la deformación plástica se produce en la viga. La rigidez en esta zona se determina a partir de la comparación de las rotaciones calculadas con los desplazamientos $\delta_{5}$ y $\delta_{6}$ con aquellas calculadas con $\delta_{3}$ y $\delta_{4}$.

\subsection{Comportamiento histerético de la conexión}

Comparando las graficas histeréticas de las Figuras 13 y 14, se observa que la conexión es capaz de soportar un ángulo de deriva de entrepiso de no menos de $0.04 \mathrm{rad}$ y experimentalmente alcanzó una rotación de $0.05 \mathrm{rad}$.

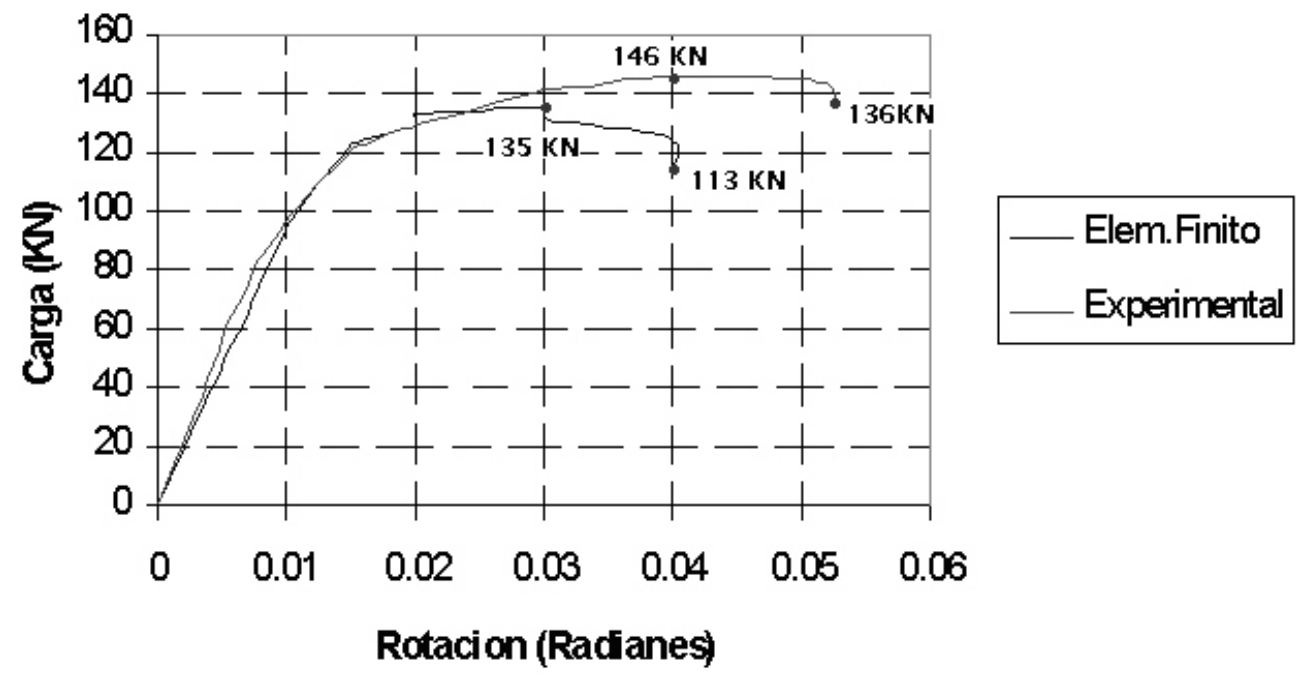

Figura 15. Comparación de carga vs. rotación entre elementos finitos y medición experimental.

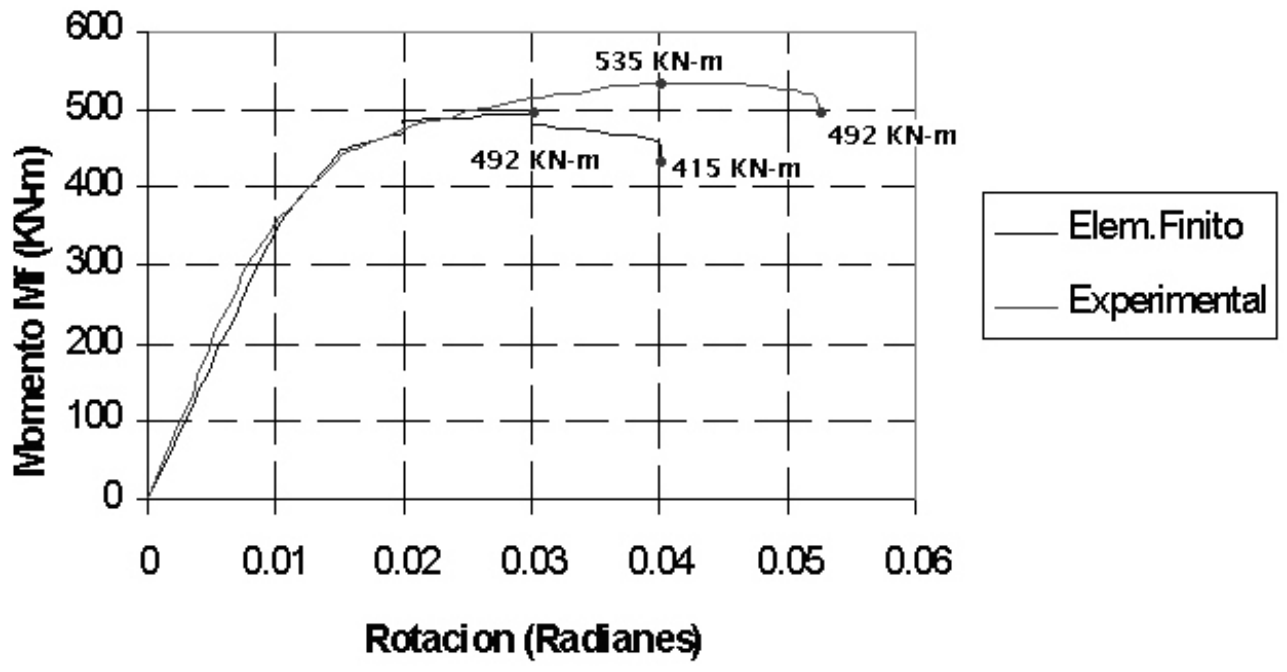

Figura 16. Comparación de momento $M_{f}$ vs. rotación entre elementos finitos ymedición experimental. 
En la Figura 14, se observa que la resistencia a flexión de la conexión en la cara de la columna $\left(M_{f}=492 \mathrm{kN}-\mathrm{m}\right.$, experimental; $M_{f}=415 \mathrm{kN}-\mathrm{m}$, elementos finitos) fue mayor que el $80 \%$ del momento plástico nominal de la viga $\left(0.8 M_{p}=259 \mathrm{kN}-\mathrm{m}\right)$.

Se observa en el gráfico experimental de las Figuras 13 y 14 que el actuador del marco de pruebas generó problemas al regreso con carga (sentido norte); sin embargo, esto no incidió para que la conexión fallara de acuerdo a lo previsto en el análisis de elementos finito ya que el comportamiento de la conexión tanto en carga como en descarga fue simétrico. También se observa en las Figuras 13 a 16 que el rango elástico se desarrolló hasta una rotación de $0.10 \mathrm{rad}$.

En las Figuras 15 y 16 se observa que hasta el ciclo de $0.025 \mathrm{rad}$, el comportamiento observado con el modelo de elementos finitos se parece al comportamiento medido experimentalmente, y para deformaciones mayores existe un desfase entre ellos.

\section{Conclusiones}

El procedimiento de diseño y el detallado de la conexión fueron exitosamente validados por el ensayo experimental de precalificación cíclica a escala real y mediante la simulación del ensayo en elementos finitos, siguiendo los lineamientos del AISC-2002.

El comportamiento de la conexión fue el esperado y logró una máxima rotación plástica en la viga de $0.05 \mathrm{rad}$ para el ensayo cíclico experimental, y de $0.04 \mathrm{rad}$ en la simulación de la prueba, sin contribución de la zona del panel de la columna a la rotación.

Los demás elementos que componen la conexión tuvieron un comportamiento elástico; no presentaron alguna concentración peligrosa de esfuerzos. El momento plástico en la cara de la columna $\left(M_{f}\right)$ supera notablemente el $80 \%$ del momento plástico nominal de la viga $\left(M_{p}\right)$.

La rótula plástica se presentó en la luz de la viga, después de la conexión a continuación de la cubre placa (como estaba calculado en el diseño) con gran fluencia y pandeo local del alma y las aletas. Lo anterior indica que la conexión cumplió con la condición de columna fuerte-viga débil, comportamiento adecuado para pórticos resistentes a momento.

El mecanismo de falla fue dúctil, comenzando por una deformación plástica en las aletas de la viga, que se propagó con el incremento de los ciclos al alma de la viga.

El detallado de la conexión precalificada se puede usar para pórticos a momento con capacidad de disipación de energía especial (DES), en regiones de alta sismicidad.

Para la conexión detallada se recomienda hacer ensayos con diferentes dimensiones de vigas y columnas, y verificar que cumple con el sistema de diseño planteado.

El uso de elementos finitos fue útil para predecir el comportamiento de la conexión, con resultados cercanos a las mediciones experimentales. Se recomienda promover el uso de esta herramienta para precalificar conexiones y así reducir los costos en materiales y laboratorio.

La aproximación del trabajo realizado proviene de un planteamiento simple y práctico, para el diseño de conexiones a momento soldadas viga-columna, planteados en publicaciones de FEMA y AISC, derivado de las observaciones realizadas en diferentes eventos sísmicos.

\section{Agradecimientos}

Los autores agradecen la asesoría permanente del profesor José Jaime García de la Universidad del Valle para la modelación matemática del prototipo, y la asesoría del profesor Luis Garza, de la Universidad Nacional, Medellín. Igualmente, agradecen al ingeniero César Freddy Gil por la donación de materiales y construcción del prototipo experimental, y por el continuo apoyo para la realización exitosa del ensayo experimental. También agradecen al personal técnico y de apoyo del laboratorio de estructuras de la Escuela de Ingeniería Civil y Geomática de la Universidad del Valle. 


\section{Referencias bibliográficas}

AISC (American Institute of Steel Construction). (2002). Seismic provisions for structural steel buildings, II. Draft. Chicago: AISC, SSPEC.

AWS (American Welding Society). (2004). AWS D1.1/D1.1M. Structural welding code-steel. 19th Edition.

Beer, P. F., Johnston, E. R., \& Dewolf, J. T. (2003). Mecánica de materiales. Tercera edición. McGraw Hill.

FEMA (Federal Emergency Management Agency). (2000). FEMA 350. Recommended seismic design criteria for new steel momentframe building.
FEMA (Federal Emergency Management Agency). (2000). FEMA 355 D. State of the art report on connection performance.

FEMA (Federal Emergency Management Agency). (2000). FEMA 355 E. State of the report on past performance of steel moment-frame buildings in earthquakes.

Garza, L., Posada, J. C., \& Pavón, C. (2004). Precalificación de una conexión soldada a momento viga-columna. Tesis de grado, Universidad Nacional de Medellín.

McCormac, J. C. (1996). Diseño de estructuras de acero. Método LRFD. México: Alfaomega Grupo Editorial. 